\title{
Erratum to: Effects of redox-active interlayer anions on the oxygen evolution reactivity of NiFe-layered double hydroxide nanosheets
}

Daojin Zhou ${ }^{1}$, Zhao Cai ${ }^{1}$, Yongmin Bi ${ }^{1}$, Weiliang Tian ${ }^{1,2}$, Ma Luo ${ }^{1}$, Qian Zhang ${ }^{1}$, Qian Zhang ${ }^{1}$, Qixian Xie ${ }^{1}$, Jindi Wang ${ }^{1}$, Yaping $\mathrm{Li}^{1}$, Yun Kuang ${ }^{1}$, Xue Duan ${ }^{1}$, Michal Bajdich ${ }^{3}$, Samira Siahrostami ${ }^{4}(\bowtie)$, and Xiaoming $\operatorname{Sun}^{1}(\bowtie)$

${ }^{1}$ State Key Laboratory of Chemical Resource Engineering, College of Energy, Beijing Advanced Innovation Center for Soft Matter Science and Engineering, Beijing University of Chemical Technology, Beijing 100029, China

${ }^{2}$ Key Laboratory of Chemical Engineering in South Xinjiang, College of Life Science, Tarim University, Alar 843300, China

${ }^{3}$ SUNCAT Center for Interface Science and Catalysis, SLAC National Accelerator Laboratory, Menlo Park, California 94025, USA

${ }^{4}$ SUNCAT Center for Interface Science and Catalysis, Department of Chemical Engineering, Stanford University, Stanford, California 94305, USA

(c) Tsinghua University Press and Springer-Verlag GmbH Germany, part of Springer Nature 2019.

\section{Erratum to}

Nano Research 2018, 11(3): 1358-1368

https://doi.org/10.1007/s12274-017-1750-9

The labels in Fig. 8 in the original version of this article were unfortunately misplaced. The corrected figure is as follow.
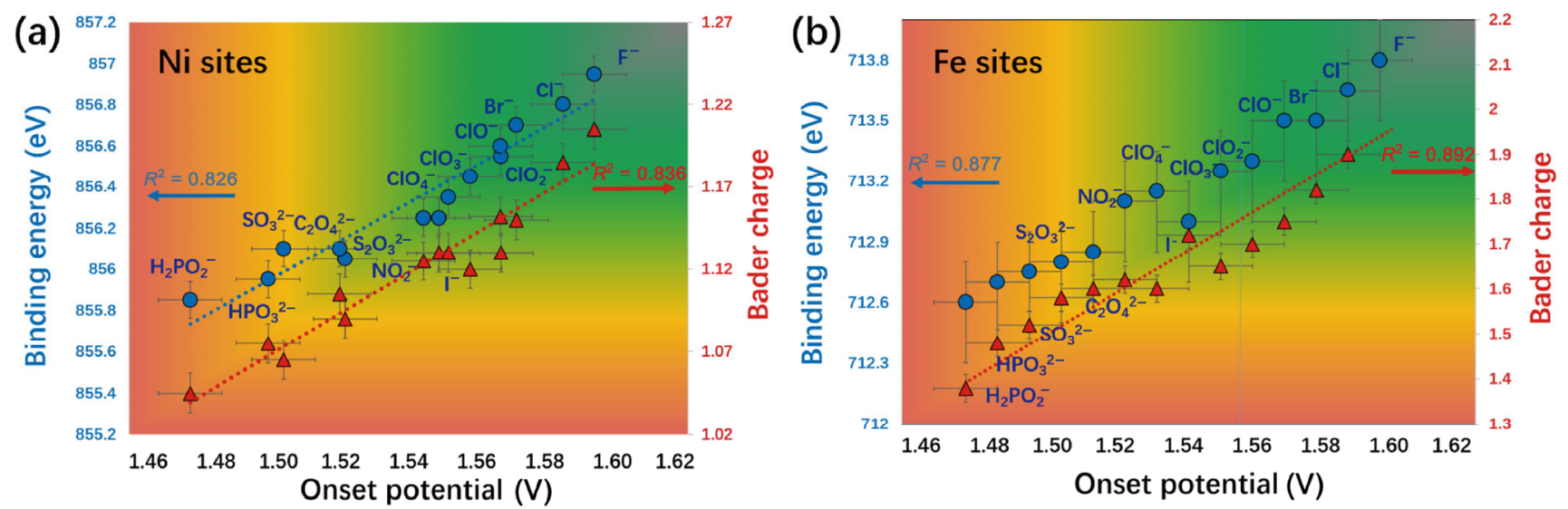

Figure 8 Binding energy and Bader charge of (a) Ni and (b) Fe sites as a function of the experimentally measured onset potential in as-prepared LDHs (O): Binding energy; $\boldsymbol{\Delta}$ : Bader charge).

The online version of the original article can be found at https://doi.org/10.1007/s12274-017-1750-9 